\title{
Does an imbalance in circulating vascular endothelial growth factors (VEGFs) cause atrial fibrillation in patients with valvular heart disease?
}

\author{
Keke Wang ${ }^{1,2}$, Yanyan Liu ${ }^{3}$, Suiqing Huang ${ }^{1,4}$, Huayang Li ${ }^{1}$, Jian Hou ${ }^{1,4}$, Jiaxing Huang ${ }^{1}$, Jiantao Chen ${ }^{1}$, \\ Kangni Feng', Mengya Liang ${ }^{1}$, Guangxian Chen ${ }^{1}$, Zhongkai $\mathrm{Wu}^{1,4}$ \\ ${ }^{1}$ Department of Cardiac Surgery, ${ }^{2}$ Department of Emergency, The First Affiliated Hospital of Sun Yat-sen University, Guangzhou 510080, China; \\ ${ }^{3}$ Department of Pathology, The First Affiliated Hospital of Traditional Medicine University, Guangzhou 510405, China; ${ }^{4}$ Key Laboratory of Assisted \\ Circulation, Ministry of Health, Sun Yat-sen University, Guangzhou 510080, China \\ Contributions: (I) Conception and design: Z Wu, K Wang, Y Liu; (II) Administrative support: J Chen, K Feng; (III) Provision of study materials \\ or patients: S Huang, J Huang; (IV) Collection and assembly of data: H Li, J Hou; (V) Data analysis and interpretation: M Liang, G Chen; (VI) \\ Manuscript writing: All authors; (VII) Final approval of manuscript: All authors. \\ Correspondence to: Zhongkai Wu. Department of Cardiac Surgery, The First Affiliated Hospital of Sun Yat-sen University, 58 Zhongshan Rd. No. 2, \\ Guangzhou 510080, China. Email: wuzhk@mail.sysu.edu.cn.
}

Background: The pathogenesis of atrial fibrillation (AF) remains unclear. Vascular endothelial growth factors (VEGFs) can stimulate fibrosis within the atrium and ventricle. We hypothesized that there is a relationship between the serum VEGFs/soluble vascular endothelial growth factor receptor (sVEGFRs) levels and $\mathrm{AF}$ in patients with valvular heart disease (VHD). This provides a new paradigm for studying AF.

Methods: The plasma levels of VEGF-A, VEGF-C, sVEGFR-1 and sVEGFR-2 were detected by enzymelinked immunosorbent assay (ELISA). A total of 100 people, consisting of AF patients (long-standing, persistent AF; $n=49)$, sinus rhythm (SR) patients $(n=31)$ and healthy controls $(n=20)$, were included in this study.

Results: The plasma levels of VEGF-A were significantly higher in AF patients compared to healthy control $(\mathrm{P}<0.05)$. The plasma levels of sVEGFR-1 were significantly higher in AF compared to $\mathrm{SR}(\mathrm{P}<0.05)$. The plasma levels of sVEGFR-2 were significantly lower in AF patients compared to SR patients and healthy controls (both $\mathrm{P}<0.05$ ). There was a significant and negative correlation between $\mathrm{AF}$ and the sVEGFR-2 levels in the groups $(\mathrm{r}=-0.432, \mathrm{P}=0.000)$.

Conclusions: An imbalance in VEGFs and sVEGFRs may contribute to AF by breaking the balance of angiogenesis and lymphangiogenesis. Additionally, sVEGFR-2 may be an important biomarker of AF.

Keywords: Atrial fibrillation (AF); vascular endothelial growth factor-A (VEGF-A); vascular endothelial growth factor-C (VEGF-C); soluble vascular endothelial growth factor receptor-1 (sVEGFR-1); soluble vascular endothelial growth factor receptor-2 (sVEGFR-2)

Submitted Jul 13, 2019. Accepted for publication Oct 31, 2019.

doi: $10.21037 /$ jtd.2019.11.32

View this article at: http://dx.doi.org/10.21037/jtd.2019.11.32

\section{Introduction}

Atrial fibrillation (AF), one of the most prevalent cardiac arrhythmias, affects $2.5-3.5 \%$ of the population worldwide, and increases the overall and cardiovascular mortality of patients $(1,2)$. Furthermore, AF is commonly associated with valvular heart disease (VHD), with VHD also present in $63.5 \%$ of AF patients in Europe (3). The outcomes of AF are not satisfactory, with neither medication nor ablation leading to sufficient resolution $(4,5)$.

The pathophysiology of AF is highly complex, and the mechanisms underlying the initiation and maintenance of $\mathrm{AF}$ remain elusive (6,7). It is well known that $\mathrm{AF}$ occurs in 
the setting of hypertension, VHD, coronary artery disease, congenital heart disease, alcohol intake, and various other conditions. It is possible that different causes of AF may share common features, or that distinct mechanisms may lead to the same symptoms. It was found that the level of vascular endothelial growth factor (VEGF) is higher in the left atrium of paroxysmal AF patients versus healthy subjects, suggesting that the heart itself is the most likely source of VEGF (8). There is evidence that VEGF and VEGF receptor (VEGFR) mRNA are both expressed in heart tissue $(9,10)$. Many pathological conditions, such as inflammation, oxygen deficiency, and pulsatile mechanical stretch, induce VEGF secretion (11). It was previously noted that elevated VEGF was often associated with angiotensin-2 (Ang-2) and von Willebrand factor (vWF), suggesting a possible link between abnormal endothelial damage/dysfunction and angiogenesis, which would contribute to the prothrombinase state in AF (12). It was also shown that VEGF could stimulate fibrosis within the atrial tissue (13). Evaluating the expression and balance between VEGFs and their receptors may represent a new paradigm in the study of AF.

The VEGF-VEGFR system is crucial in angiogenesis and lymphangiogenesis (14). VEGFs activate VEGFR tyrosine kinases in endothelial cells to stimulate angiogenesis and lymphangiogenesis (15). There are five VEGF family members: VEGF-A through D and placenta growth factor (PlGF). Their regulation of angiogenesis mainly depends on binding to VEGFR-1 and VEGFR-2 (16). There are also two soluble VEGFRs, sVEGFR-1 and sVEGFR-2, which can act as negative regulators of the VEGF system by trapping VEGF family ligands such as VEGF-A, VEGF-C and VEGF-D (17).

We hypothesized that the VEGFs/sVEGFRs play an important role in the progression and maintenance of $\mathrm{AF}$. In order to explore this hypothesis, we measured the plasma levels of VEGF-A, VEGF-C, sVEGFR-1 and sVEGFR-2 in VHD patients with persistent AF.

\section{Methods}

\section{Clinical information of participants}

We recruited 80 patients with VHD who were admitted to the Department of Cardiac Surgery of The First Affiliated Hospital of Sun Yat-sen University in China between March 2015 and March 2017. Forty-nine VHD patients had persistent AF. Persistent AF was defined as AF that had persisted without interruption for 7 days or longer in patients receiving pharmacological intervention, or for whom electrical cardioversion was required to terminate the arrhythmia. Although there was some variation among patients, the disease severity was generally similar as assessed by their history of AF (all longer than 1 year) and the ultrasound findings. A total of 31 patients had sinus rhythm (SR). All of the patients had VHD, underwent a valve replacement procedure, and recovered well. We also recruited 20 subjects without VHD or any history of AF as a healthy control group. Each patient's heart rhythm was determined by an electrocardiogram taken at the time of admission. VHD was diagnosis by ultrasonic cardiogram and a post-operation pathological examination.

The exclusion criteria included recent-onset acute cardiovascular or cerebrovascular events, trauma or surgery, coronary artery heart disease, malignancy, connective tissue disease, acute or chronic infection, and pulmonary, hepatic, or renal dysfunction. Patients who were receiving treatment with anti-inflammatory or steroid drugs were also excluded. A formal medical and drug history-taking was done for each enrolled patient. All patients received a physical examination and routine laboratory tests. This study was approved by the Ethics Committee of The First Affiliated Hospital of Sun Yat-sen University, and all patients enrolled in this study gave written informed consent.

\section{Blood samples and biomarker analysis}

Blood samples from both VHD patients and controls were obtained from the forearm veins using $6.0 \mathrm{~mL}$ vacutainer tubes (BD, Plymouth, UK) containing EDTA. The blood samples were immediately placed on ice before being centrifuged at 3,000 rpm for 10 minutes at $4{ }^{\circ} \mathrm{C}$. Plasma samples were then stored at $-80^{\circ} \mathrm{C}$ until analysis. The plasma levels of sVEGFR-1, sVEGFR-2, VEGF-A, and VEGF-C were determined by enzyme-linked immunosorbent assays (Otsuka Pharmaceutical Co. Ltd., Tokyo, Japan).

\section{Statistical analysis}

Values are expressed as the mean [ \pm standard deviation $(\mathrm{SD})]$ numbers and percentages. Categorical data were compared using a chi-squared test, and two-sample $t$-tests and MannWhitney tests were used as appropriate for parametric and non-parametric continuous variables. A receiver-operating characteristic (ROC) curve analysis was used to calculate the area under the curve (AUC) for the ability of the variables 
Table 1 Clinical characteristics of the study groups

\begin{tabular}{lccr}
\hline Parameters & Control $(\mathrm{n}=20)$ & VHD with SR $(\mathrm{n}=31)$ & VHD with AF $(\mathrm{n}=49)$ \\
\hline Age $($ years $)$ & $32( \pm 9)$ & $56( \pm 11)$ & $55( \pm 10)$ \\
Men, $\mathrm{n}(\%)$ & $9(45.0)$ & $26(83.9)$ & $14(28.6)$ \\
BMI $\left(\mathrm{kg} / \mathrm{m}^{2}\right)$ & $21.6( \pm 3.6)$ & $23.0( \pm 2.5)$ & $22.9( \pm 3.7)$ \\
Hypertension, $\mathrm{n}(\%)$ & $0(0.0)$ & $10(32.3)$ & $3(6.1)$ \\
Diabetes mellitus, $\mathrm{n}(\%)$ & $0(0.0)$ & $3(9.7)$ & $1(2.0)$ \\
Smokers, $\mathrm{n}(\%)$ & $0(0.0)$ & $9(22.6)$ & $8(16.3)$ \\
$\beta$-blocker treatment, $\mathrm{n}(\%)$ & $0(0.0)$ & $4(12.9)$ & $10(20.4)$ \\
Statin treatment, $\mathrm{n}(\%)$ & $0(0.0)$ & $67( \pm 8)$ & $5(10.2)$ \\
Left ventricular EF, $\mathrm{n}(\%)$ & $68( \pm 7)$ & $83.1( \pm 25.7)$ & $60( \pm 8)^{\star *}$ \\
Creatinine & $82.1( \pm 24.3)$ & $22.4( \pm 13.3)$ & $83.7( \pm 23.3)$ \\
ALT & $21.4( \pm 14.6)$ & $899.1( \pm 1,396.7)$ & $28.6( \pm 8.5)$ \\
NT-proBNP & $435.1( \pm 876.4)$ & $1,319.9( \pm 1,002.2)$ & \\
\hline
\end{tabular}

The results are expressed as numbers (percentages) or means ( \pm standard deviation). Chi-squared values were used for categorical data, and two-sample $t$-tests and Mann-Whitney tests were used (as appropriate) for parametric and non-parametric continuous variables. ${ }^{*}$, $\mathrm{P}<0.001$, SR vs. AF. VHD, valvular heart disease; SR, sinus rhythm; AF, atrial fibrillation; BMl, body mass index; $E F$, ejection fraction; $A L T$, alanine aminotransferase; NT-proBNP, N-terminal pro-brain natriuretic peptide.

to predict $\mathrm{AF}$ in VHD patients. Multiple logistic regression models were also performed to assess the ability to predict $\mathrm{AF}$ in VHD patients. Comparisons between groups were made using a one-way analysis of variance (one-way ANOVA). Correlations were determined using Pearson's correlation or Spearman's rank correlation method, as appropriate. A two-tailed $\mathrm{P}$ value $<0.05$ was considered statistically significant. Statistical analyses were performed with IBM SPSS 25.0 for Windows (IBM SPSS, Armonk, NY, USA).

\section{Results}

\section{Baseline characteristics}

The demographic and clinical characteristics of the study population, together with the laboratory findings, are presented in Table 1. The mean age, number of smokers, number of subjects with a history of hypertension or diabetes, and number of subjects receiving treatment with $\beta$-blockers or statins were significantly higher in the two groups of VHD patients than in the controls. The percentage of males and mean body mass index were not significantly different between the VHD patients and controls.

There were no significant differences between the SR and $\mathrm{AF}$ groups in terms of the sex ratio, mean body mass index, smoking status, history of hypertension or diabetes, treatment with $\beta$-blockers or statins, or in the creatinine and N-terminal pro-brain natriuretic peptide (NT-proBNP) levels. The left ventricular ejection fraction (EF) (\%) was significantly higher in the SR patients than in the AF patients, which was expected given that $\mathrm{AF}$ can influence the ventricular systolic function. The alanine aminotransferase (ALT) level was significantly higher in the AF patients than in the SR patients. This may have been due to the decreased cardiac function and increased pulmonary circulatory pressure, which might have resulted in hepatic congestion.

\section{The concentrations of VEGFs among the AF, SR and bealthy control subjects}

The differences in the serum VEGF-A and VEGF-C levels among patients with $\mathrm{AF}$ and SR and controls are shown in Table 2. The plasma levels of VEGF-A were significantly higher in AF patients than in healthy controls [mean 188.81 (95\% CI: 121.99-255.63) versus 88.90 (95\% CI: $65.52-$ 112.28) $\mathrm{pg} / \mathrm{mL}, \mathrm{P}=0.02$ by one-way ANOVA]. The plasma levels of VEGF-A were also higher in SR patients than in healthy controls [mean 149.37 (95\% CI: 96.10-202.63) versus 88.90 (95\% CI: $65.52-112.28) \mathrm{pg} / \mathrm{mL}, \mathrm{P}=0.23$ by 
Table 2 The VEGF-sVEGFR levels in the three study groups

\begin{tabular}{|c|c|c|c|c|c|}
\hline Parameter & Control $(n=20)$ & VHD with $S R(n=31)$ & $P$ value $^{a}$ & VHD with AF $(n=49)$ & $P$ value ${ }^{b}$ \\
\hline VEGF-C (pg/mL) & $1,040.20( \pm 308.61)$ & $956.56( \pm 510.92)$ & 0.40 & $1,051.91( \pm 543.35)$ & 0.93 \\
\hline sVEGFR-1 (pg/mL) & $92.91( \pm 26.64)$ & $82.77( \pm 41.85)$ & 0.03 & $138.87( \pm 149.48)$ & 0.11 \\
\hline sVEGFR-2 (pg/mL) & $6,239.05( \pm 1,030.40)$ & $5,351.81( \pm 1,150.83)$ & 0.00 & $4,627.65( \pm 1,076.69)$ & 0.00 \\
\hline
\end{tabular}

The results are expressed as the means ( \pm standard deviation). Two-sample $t$-tests and Mann-Whitney tests were used as appropriate for parametric and non-parametric continuous variables. Comparisons between groups were made using a one-way Analysis of Variance (one-way ANOVA). P value : atrial fibrillation vs. sinus rhythm; $\mathrm{P}$ value ${ }^{\mathrm{b}}$ : atrial fibrillation vs. control. VEGF, vascular endothelial growth factor; sVEGFR, soluble vascular endothelial growth factor receptor; VHD, valvular heart disease; SR, sinus rhythm; AF, atrial fibrillation.

one-way ANOVA]. The plasma levels of VEGF-A were not significantly different between the AF and SR patients. The area under the ROC curve for VEGF-A was 0.57 (95\% CI: $0.42-0.71)$. There were no significant differences among the AF, SR and healthy control groups in terms of the plasma levels of VEGF-C. The area under the ROC curve for VEGF-C was 0.50 (95\% CI: 0.39-0.62).

\section{The concentrations of soluble VEGFRs among the subjects}

The plasma levels of sVEGFR-1 were significantly higher in $\mathrm{AF}$ patients than SR patients [mean 138.87 (95\% CI: 92.87-184.88) versus 82.77 (95\% CI: 66.85-98.69) $\mathrm{pg} / \mathrm{mL}$, $\mathrm{P}=0.03$ by one-way ANOVA]. The plasma levels of sVEGFR-1 were also higher in AF patients than healthy control [mean 138.87 (95\% CI: 92.87-184.88) versus 92.91 (95\% CI: $80.44-105.38) \mathrm{pg} / \mathrm{mL}, \mathrm{P}=0.11$ by one-way ANOVA]. However, there was no statistically significant difference between the SR group and healthy controls. The area under the ROC curve for sVEGFR-1 was 0.65 (95\% CI: 0.53-0.76).

The plasma levels of sVEGFR-2 were highest in the healthy controls, followed by the SR group, then the AF group. The plasma levels of sVEGFR-2 were significantly lower in the AF group than in the SR group [mean 4,627.65 (95\% CI: 4,318.39-4,936.91) versus 5,351.81 (95\% CI: $4,929.68-5,773.94) \mathrm{pg} / \mathrm{mL}, \mathrm{P}=0.00$ by one-way ANOVA]. The plasma levels of sVEGFR-2 were also significantly lower in SR patients than in healthy controls [mean 5,351.81 (95\% CI: 4,929.68-5,773.94) versus 6,239.05 (95\% CI: 5,756.81-6,721.29) pg/mL, $\mathrm{P}=0.01$ by one-way ANOVA]. The area under the ROC curve for sVEGFR-2 was 0.77 (95\% CI: 0.67-0.86).

A multiple logistic regression analysis including VEGF-A, sVEGFR-1 and sVEGFR-2 was performed, and the area under the ROC curve was 0.80 (95\% CI: 0.69-0.91]. The Chi-squared test gave a value of $\mathrm{P}=0.00$.

\section{Correlation of the VEGF-A, sVEGFR-1 and SVEGFR-2 levels with other clinical features}

Correlations between and among the levels of VEGF-A, sVEGFR-1, and sVEGFR-2, the presence of AF, the left ventricular EF (\%), and the creatinine, ALT, and NTproBNP levels were examined (listed in Table 3). There was a significant positive correlation between sVEGFR-2 and the left ventricular EF ( $\mathrm{r}=0.311, \mathrm{P}=0.005)$. There was also a significant and negative correlation between $\mathrm{AF}$ and the sVEGFR-2 level $(\mathrm{r}=-0.432, \mathrm{P}=0.000)$. In addition, there was a negative correlation between the NT-proBNP and sVEGFR-2 levels ( $\mathrm{r}=-0.256, \mathrm{P}=0.024)$.

\section{Discussion}

The main findings of the present study are that the plasma levels of VEGF-A and sVEGFR-1 were significantly higher in AF patients than controls and SR patients. The plasma levels of sVEGFR-2 were significantly lower in the AF group than in the control and SR groups. The plasma levels of sVEGFR-2 were also significantly lower in the SR group than in the control group. The left ventricular EF (\%) was significantly higher in the SR patients than in the AF patients, which is in agreement with the fact that $\mathrm{AF}$ can influence the ventricular systolic function. There was a significant negative correlation between $\mathrm{AF}$ and the sVEGFR-2 level.

\section{Why are the plasma levels of VEGF-A and SVEGFR-1 significantly elevated in AF patients?}

This is the first study to demonstrate that there are 
Table 3 Correlation of the VEGF-A, sVEGFR-1 and sVEGFR-2 levels with clinical variables

\begin{tabular}{|c|c|c|c|c|c|c|}
\hline Features & \multicolumn{2}{|c|}{ VEGF-A } & \multicolumn{2}{|c|}{ sVEGFR-1 } & \multicolumn{2}{|c|}{ sVEGFR-2 } \\
\hline VEGF-A & 1.000 & - & 0.153 & 0.128 & 0.091 & 0.368 \\
\hline sVEGFR-1 & 0.153 & 0.128 & 1.000 & - & -0.071 & 0.483 \\
\hline sVEGFR-2 & 0.091 & 0.368 & -0.071 & 0.483 & 1.000 & - \\
\hline Left ventricular EF (\%) & -0.051 & 0.655 & -0.077 & 0.497 & 0.311 & 0.005 \\
\hline Creatinine & 0.005 & 0.966 & 0.004 & 0.970 & -0.007 & 0.954 \\
\hline ALT & 0.021 & 0.865 & 0.049 & 0.669 & -0.064 & 0.570 \\
\hline NT-proBNP & -0.029 & 0.805 & 0.002 & 0.985 & -0.256 & 0.024 \\
\hline
\end{tabular}

Correlations were ascertained with Pearson's correlation or Spearman's rank correlation method, as appropriate. VEGF, vascular endothelial growth factor; sVEGFR, soluble vascular endothelial growth factor receptor; EF, ejection fraction; ALT, alanine aminotransferase; NT-proBNP, N-terminal pro-brain natriuretic peptide.

differences in the plasma VEGF-A and sVEGFR-1 levels in VHD patients with and without AF. It is known that AF can cause endothelial dysfunction (18). Our results showing that the plasma levels of VEGF-A and sVEGFR-1 were significantly higher in AF patients suggests that endothelial dysfunction may be the cause of AF. The level of $\mathrm{vWF}$, an indicator of endothelial damage or dysfunction, was previously found to be increased in $\mathrm{AF}$ patients, supporting the role of endothelial dysfunction in $\operatorname{AF}(19,20)$. Further, the proliferation of circulating endothelial progenitor cells (EPCs), which contribute to endothelium repair by replacing damaged endothelial cells, was significantly reduced in patients with permanent AF (21). Electrical remodeling, structural remodeling caused by aging, coronary artery disease, hypertensive heart disease, diabetes mellitus and inflammation have all been suggested to play important roles in the progression and maintenance of AF $(22,23)$. The remodeling can be seen as atrial fibrosis (24). Further, many studies showed significantly increased secretion of VEGF, possibly originating from cardiac myocytes $(8,12,25,26)$.

Hypoxia is known to upregulate VEGF via HIF-1 $\alpha$ (27). AF can cause tissue hypoxia, and VEGF may ameliorate myocardial ischemia $(28,29)$. Using left atrial appendage (LAA) samples obtained from $59 \mathrm{AF}$ patients, Abe et al. (30) found that overexpression of HIF- $1 \alpha$ may be involved in atrial myocardial fibrosis. Scridon et al. suggested that the secretion of VEGF in the left atrium is a transient event in the natural history of $\mathrm{AF}$, where the spreading cardiac fibrosis would reduce the degree of pulsatile stretch and subsequently diminish the VEGF levels (8).

Although VEGF does not appear to play a direct role in angiogenesis or the vascular permeability in $\mathrm{AF}$, some studies had shown that VEGF could stimulate the fibrosis process within the atrium and ventricle (31-34). It is known that soluble VEGFR-1 (sFlt-1) is an endogenous VEGF inhibitor. For example, sVEGFR-1 neutralizes VEGF-A, and is critical for maintaining the corneas devoid of blood vessels in the eyes (35). According to the literature, the sVEGFR-1 level is correlated with morbidity and mortality, and is a potent marker of disease severity in patients with sepsis or who are critically-ill (36). Our findings show that the plasma levels of VEGF-A and sVEGFR-1 are both increased in AF patients. While VEGF-A promotes angiogenesis, sVEGFR-1 can downregulate VEGFR signaling, mainly by trapping VEGF-A. This system may explain why AF is associated with endothelial dysfunction and fibrosis. However, more research is needed to explain why the expression levels of sVEGFR-1 are increased in patients with persistent $\mathrm{AF}$.

The two most important complications of $\mathrm{AF}$ are thromboembolism and heart failure. In our study, we found that the plasma levels of VEGF-A and sVEGFR-1 were significantly elevated in AF patients, while the left ventricular EF (\%) was significantly higher in the $\mathrm{SR}$ patients than the AF patients. Heart failure and AF often coexist and form a vicious cycle. Risk factors such as hypertension, diabetes and valvular disease may lead 
to $\mathrm{AF}$, while $\mathrm{AF}$ can cause heart failure. However, heart failure is also a risk factor for $\mathrm{AF}$, and the incidence of $\mathrm{AF}$ is directly related to the New York Heart Association (NYHA) classification. The incidence of AF in NYHA class I heart failure patients was less than $10 \%$, while in NYHA $\mathrm{IV}$ patients, $\mathrm{AF}$ was present in more than $55 \%$ of cases. Moreover, severe heart failure can also increase the rate of AF (37-39). Our study shows that targeting VEGF-A and soluble VEGFR-1 may provide a new way to study the causes of AF, and may represent targets for treatment.

\section{Why are the plasma levels of sVEGFR-2 decreased in AF patients?}

The VEGF family plays a crucial role in the formation of blood vessels and in lymphangiogenesis (40). Atrial tissue fibrosis is promoted by lymphangiogenesis. Although VEGF-C is one of the most important factors involved in lymphangiogenesis, our results showed that the plasma levels of VEGF-C were not significantly different among the groups. sVEGFR-2 plays a key role in maintaining the status of the cornea as a lymphatic-free tissue, and it selectively inhibits lymphangiogenesis, but not angiogenesis (41). By inhibiting lymphangiogenesis, sVEGFR-2 can also influence tumor progression by regulating lymphatic formation and metastasis in vivo (42-44). However, it is unknown whether sVEGFR-2 can inhibit lymphangiogenesis in atrial tissues during fibrosis. In the present study, the plasma level of sVEGFR-2 was decreased in AF patients. This may suggest that the suppression of lymphangiogenesis was weakened in these patients. This is consistent with the finding by Berntsson et al. that increased VEGF-D is associated with AF (45). Further studies will be needed to determine whether this is the case, and to identify the detailed mechanism(s) underlying this phenomenon.

\section{Limitations}

This study is limited by its cross-sectional design. Thus, as an observational study, cause-effect relationships could not be established. Further prospective studies are necessary to confirm our present findings. The sample size of our study was also small, and did not include patients with isolated AF who didn't have VHD. As a result, we were unable to rule out the effects of VHD on the findings. We also did not evaluate the changes in the plasma levels of sVEGFR-1, sVEGFR-2, VEGF-A and VEGF-C after valve replacement surgery. Future studies might examine whether the expression of these proteins is changed after surgery, and how the levels correlate with the response to valve replacement.

\section{Conclusions}

The present findings indicate that an imbalance in the VEGFs/sVEGFRs may contribute to AF, possibly by breaking the balance between angiogenesis and lymphangiogenesis. Although further studies are needed due to the small sample size and cross-sectional design of the present study, our findings suggest that these proteins may be involved in AF, and sVEGFR-2 may represent an important biomarker of AF.

\section{Acknowledgments}

Funding: We thank the fund support of the National Key R\&D Program of China (grant number 2017YFC1105000) and the National Natural Science Foundation of China (grant number 81770319, 81570039).

\section{Footnote}

Conflicts of Interest: The authors have no conflicts of interest to declare.

Ethical Statement: The authors are accountable for all aspects of the work in ensuring that questions related to the accuracy or integrity of any part of the work are appropriately investigated and resolved. This study was approved by the Ethics Committee of The First Affiliated Hospital of Sun Yat-sen University \{No. [2017]157\}, and all patients enrolled in this study gave written informed consent.

\section{References}

1. Andersson T, Magnuson A, Bryngelsson IL, et al. All-cause mortality in 272,186 patients hospitalized with incident atrial fibrillation 1995-2008: a Swedish nationwide longterm case-control study. Eur Heart J 2013;34:1061-7.

2. Rogers PA, Bernard ML, Madias C, et al. Current Evidence-Based Understanding of the Epidemiology, Prevention, and Treatment of Atrial Fibrillation. Curr Probl Cardiol 2018;43:241-83.

3. Di Biase L. Use of Direct Oral Anticoagulants in Patients 
With Atrial Fibrillation and Valvular Heart Lesions. J Am Heart Assoc 2016. doi: 10.1161/JAHA.115.002776.

4. Berger WR, Meulendijks ER, Limpens J, et al. Persistent atrial fibrillation: A systematic review and meta-analysis of invasive strategies. Int J Cardiol 2019;278:137-43.

5. Salcedo J, Hay JW, Lam J. Cost-effectiveness of rivaroxaban versus warfarin for treatment of nonvalvular atrial fibrillation in patients with worsening renal function. Int J Cardiol 2019;282:53-8.

6. Heijman J, Voigt N, Nattel S, et al. Cellular and molecular electrophysiology of atrial fibrillation initiation, maintenance, and progression. Circ Res 2014;114:1483-99.

7. Nishida K, Nattel S. Atrial fibrillation compendium: historical context and detailed translational perspective on an important clinical problem. Circ Res 2014;114:1447-52.

8. Scridon A, Morel E, Nonin-Babary E, et al. Increased intracardiac vascular endothelial growth factor levels in patients with paroxysmal, but not persistent atrial fibrillation. Europace 2012;14:948-53.

9. Levy AP, Levy NS, Loscalzo J. Regulation of vascular endothelial growth factor in cardiac myocytes. Circ Res 1995;76:758-66.

10. Seko Y, Seko Y, Takahashi N, et al. Pulsatile stretch stimulates vascular endothelial growth factor (VEGF) secretion by cultured rat cardiac myocytes. Biochem Biophys Res Commun 1999;254:462-5.

11. Li J, Hampton T, Morgan JP, et al. Stretch-induced VEGF expression in the heart. J Clin Invest 1997;100:18-24.

12. Freestone B, Chong AY, Lim HS, et al. Angiogenic factors in atrial fibrillation: a possible role in thrombogenesis? Ann Med 2005;37:365-72.

13. Chung CC, Kao YH, Yao CJ, et al. A comparison of left and right atrial fibroblasts reveals different collagen production activity and stress-induced mitogen-activated protein kinase signalling in rats. Acta Physiol (Oxf) 2017;220:432-45.

14. Wang Y, Nakayama M, Pitulescu ME, et al. Ephrin-B2 controls VEGF-induced angiogenesis and lymphangiogenesis. Nature 2010;465:483-6.

15. Tammela T, Zarkada G, Wallgard E, et al. Blocking VEGFR-3 suppresses angiogenic sprouting and vascular network formation. Nature 2008;454:656-60.

16. Rahimi N. VEGFR-1 and VEGFR-2: two nonidentical twins with a unique physiognomy. Front Biosci 2006;11:818-29.

17. Albuquerque RJ. The newest member of the VEGF family. Blood 2013;121:4015-6.

18. Takahashi N, Ishibashi Y, Shimada T, et al. Atrial fibrillation impairs endothelial function of forearm vessels in humans. J Card Fail 2001;7:45-54.

19. Li-Saw-Hee FL, Blann AD, Gurney D, et al. Plasma von Willebrand factor, fibrinogen and soluble P-selectin levels in paroxysmal, persistent and permanent atrial fibrillation. Effects of cardioversion and return of left atrial function. Eur Heart J 2001;22:1741-7.

20. Mondillo S, Sabatini L, Agricola E, et al. Correlation between left atrial size, prothrombotic state and markers of endothelial dysfunction in patients with lone chronic nonrheumatic atrial fibrillation. Int J Cardiol 2000;75:227-32.

21. Tan Q, Zhang S, Qi X, et al. Permanent atrial fibrillation impairs the function of circulating endothelial progenitor cells. Postgrad Med 2017;129:198-204.

22. Allessie M, Ausma J, Schotten U. Electrical, contractile and structural remodeling during atrial fibrillation. Cardiovasc Res 2002;54:230-46.

23. Lau DH, Nattel S, Kalman JM, et al. Modifiable Risk Factors and Atrial Fibrillation. Circulation 2017;136:583-96.

24. Thiedemann KU, Ferrans VJ. Left atrial ultrastructure in mitral valvular disease. Am J Pathol 1977;89:575-604.

25. Seko Y, Nishimura H, Takahashi N, et al. Serum levels of vascular endothelial growth factor and transforming growth factor-beta 1 in patients with atrial fibrillation undergoing defibrillation therapy. Jpn Heart J 2000;41:27-32.

26. Ogi H, Nakano $Y$, Niida $S$, et al. Is structural remodeling of fibrillated atria the consequence of tissue hypoxia? Circ J 2010;74:1815-21.

27. Jain T, Nikolopoulou EA, Xu Q, et al. Hypoxia inducible factor as a therapeutic target for atherosclerosis. Pharmacol Ther 2018;183:22-33.

28. Wong AK, Marais HJ, Jutzy K, et al. Isolated atrial infarction in a patients with single vessel disease of the sinus node artery. Chest 1991;100:255-6.

29. Jesel L, Abbas M, Toti F, et al. Microparticles in atrial fibrillation: a link between cell activation or apoptosis, tissue remodelling and thrombogenicity. Int J Cardiol 2013;168:660-9.

30. Abe I, Teshima Y, Kondo H, et al. Association of fibrotic remodeling and cytokines/chemokines content in epicardial adipose tissue with atrial myocardial fibrosis in patients with atrial fibrillation. Heart Rhythm 2018;15:1717-27.

31. Hamada N, Kuwano K, Yamada M, et al. Anti- 
Vascular Endothelial Growth Factor Gene Therapy Attenuates Lung Injury and Fibrosis in Mice. J Immunol 2005; 175:1224-31.

32. Chaudhary NI, Roth GJ, Hilberg F, et al. Inhibition of PDGF, VEGF and FGF signalling attenuates fibrosis. Eur Respir J 2007;29:976-85.

33. Pimentel RC, Yamada KA, Kléber AG, et al. Autocrine regulation of myocyte $\mathrm{Cx} 43$ expression by VEGF. Circ Res 2002;90:671-7.

34. Yamada K, Green KG, Samarel AM, et al. Distinct pathways regulate expression of cardiac electrical and mechanical junction proteins in response to stretch. Circ Res 2005;97:346-53.

35. Ambati BK, Nozaki M, Singh N, et al. Corneal avascularity is due to soluble VEGF receptor-1. Nature 2006;443:993-7.

36. Pal E, Korva M, Resman Rus K, et al. Relationship between circulating vascular endothelial growth factor and its soluble receptor in patients with hemorrhagic fever with renal syndrome. Emerg Microbes Infect 2018;7:89.

37. Morin DP, Bernard ML, Madias C, et al. The State of the Art: Atrial Fibrillation Epidemiology, Prevention, and Treatment. Mayo Clin Proc 2016;91:1778-810.

38. Staerk L, Sherer JA, Ko D, et al. Atrial Fibrillation: Epidemiology, Pathophysiology, and Clinical Outcomes. Circ Res 2017;120:1501-17.

39. Gillis AM. Atrial Fibrillation and Ventricular Arrhythmias:
Sex Differences in Electrophysiology, Epidemiology, Clinical Presentation, and Clinical Outcomes. Circulation 2017;135:593-608.

40. Shibuya M. Vascular endothelial growth factor and its receptor system: physiological functions in angiogenesis and pathological roles in various diseases. J Biochem 2013;153:13-9.

41. Maehana S, Nakamura M, Ogawa F, et al. Suppression of lymphangiogenesis by soluble vascular endothelial growth factor receptor-2 in a mouse lung cancer model. Biomed Pharmacother 2016;84:660-5.

42. Albuquerque RJ, Hayashi T, Cho WG, et al. Alternatively spliced vascular endothelial growth factor receptor-2 is an essential endogenous inhibitor of lymphatic vessel growth. Nat Med 2009;15:1023-30.

43. Becker J, Pavlakovic H, Ludewig F, et al. Neuroblastoma progression correlates with downregulation of the lymphangiogenesis inhibitor sVEGFR-2. Clin Cancer Res 2010;16:1431-41.

44. Shibata MA, Ambati J, Shibata E, et al. The endogenous soluble VEGF receptor-2 isoform suppresses lymph node metastasis in a mouse immunocompetent mammary cancer model. BMC Med 2010;8:69.

45. Berntsson J, Smith JG, Johnson LSB, et al. Increased vascular endothelial growth factor $\mathrm{D}$ is associated with atrial fibrillation and ischaemic stroke. Heart 2019;105:553-8.
Cite this article as: Wang K, Liu Y, Huang S, Li H, Hou J, Huang J, Chen J, Feng K, Liang M, Chen G, Wu Z. Does an imbalance in circulating vascular endothelial growth factors (VEGFs) cause atrial fibrillation in patients with valvular heart disease? J Thorac Dis 2019;11(12):5509-5516. doi: 10.21037/ jtd.2019.11.32 